\title{
Immunohistochemical Localization of Osteopontin - A Comparative Study on Periodontally Healthy and Diseased Tooth Root Surface
}

\author{
Julie Toby Thomas ${ }^{1}$, Toby Thomas ${ }^{2}$ \\ ${ }^{1}$ Department of Periodontology, Zulfi Dental College, Female section, Majma University, Saudi Arabia \\ ${ }^{2}$ Department of Conservative Dentistry, Zulfi Dental College, Female section, Majma University, Saudi Arabia
}

Corresponding author: Julie Toby Thomas, Department of Periodontology, Zulfi Dental College, Female Section, Majma University, Saudi Arabia, Tel: 0164044305; E-mail: drthomastoby@gmail.com

Received date: September 20, 2017; Accepted date: October 03, 2017; Published date: October 10, 2017

Copyright: (C) 2017 Thomas JT. This is an open-access article distributed under the terms of the creative Commons Attribution License, which permits unrestricted use, distribution and reproduction in any medium, provided the original author and source are credited.

Citation: Thomas JT. Immunohistochemical Localization of Osteopontin - A Comparitive Study on Periodontally Healthy and Diseased Tooth Root Surface. Periodon Prosthodon. 2017, Vol.3 No.2:11.

\section{Abstract}

A Great deal of effort has been focused on trying to determine the presence and localization of factors responsible for cementogenesis, as well as in repair and regeneration of periodontal tissues. Bone sialoprotein and osteopontin are the two major non-collagenous proteins in cementum that appeared to have important roles in cementogenesis and regeneration.

Aim: To investigate the expression and distribution of osteopontin on diseased root surface in comparison with healthy non-diseased root surface in by immunohistochemical technique using osteopontin mouse monoclonal antibody [NCL-O-PONTIN] (Novocastra)

Materials and Methods: Fifty-Five teeth which included 25 permanent healthy human teeth and 30 permanent teeth affected by advanced periodontitis were collected. All teeth were initially fixed in $10 \%$ neutral buffered formalin. Following fixation and demineralization, specimens were embedded in paraffin, sectioned and exposed to antibodies against osteopontin. Stained sections were assessed using digital photomicroscope.

Results: In healthy root surface staining intensity was found to be mild to moderate in the cemental surface and matrix. Moderate staining was found in relation to periodontal ligament. In diseased root surface there was absence of immunostaining for osteopontin (OPN) in exposed cementum. In, the apical part of the diseased tooth, a strong staining for OPN was seen along the cementum surfaces adjacent to the periodontal ligament.

Conclusion: The absence of staining for OPN in all areas of exposed cementum (absence of overlying periodontal ligament) in diseased teeth can affect the ability for regeneration and new connective tissue attachment onto the previously denuded root surface.
Keywords: Osteopontin, Diseased Tooth, OPN, Periodontal ligament

\section{Introduction}

Periodontitis is an inflammatory disease of the supporting tissues of the teeth caused by specific microorganisms or groups of specific microorganisms resulting in progressive destruction of the periodontal ligament and alveolar bone with pocket formation, recession or both. Hence damage occurs to the tooth supporting tissues, resulting in exposure of the root surface to the oral environment [1].

Periodontitis is manifested clinically as continued bleeding on probing and clinical attachment loss, in addition to changes in colour, contour and consistency. Host mediated tissue destruction occurs via complement activation, release of lysosomal enzymes and connective tissue matrix metalloproteinases. Microbial enzymes may damage connective tissue directly, and together with toxic metabolites and structural materials, are thought to disrupt the reparative activities of fibroblasts and cells of the immune defences.

Cementum was first described by Denton, as a calcified, avascular mesenchymal tissue that forms the outer covering of the anatomic root [2]. Cementum approximately comprises of $50 \%$ hydroxyapatite and $50 \%$ organic matrix which consists of collagen and non-collagenous proteins. Protein extracts of mature cementum promote cell attachment, migration and stimulate protein synthesis of gingival fibroblast and periodontal ligament cells [3].

One of the main functions of cementum is to anchor the principal collagen fibres of the periodontal ligament to the root surface. Cementum also has important adaptive and reparative functions, playing a crucial role to maintain the occlusal relationship and to protect the integrity of the root surface. Regeneration of cementum on denuded root surface has been the subject of interest in the modern era of periodontal plastic surgery. Studies conducted by Linskog et al. 
[4], suggested the inability of the periodontium to regenerate without the presence of cementum.

Hence, a great deal of effort has been focused on trying to determine the presence and localization of factors responsible for cementogenesis, as well as in repair and regeneration of periodontal tissues. Bone sialoprotein and osteopontin are the two major non-collagenous proteins in cementum that appeared to have important roles in cementogenesis and regeneration. Both are phosphorylated and sulfated glycoproteins that are prominently expressed in acellular extrinsic fiber cementum and acellular afibrillar cementum. They are expressed during early tooth root development by cells along the root surface [3].

Osteopontin is predominantly seen within the periodontal ligament region of mature teeth. They play a major role in the differentiation of the cementoblast progenitor cells to cementoblasts. The phosphoglycoprotein osteopontin contains the well-recognized adhesion domain, arginine- glycineaspartic acid (RGD) which targets specific intergrin receptors, as well as other adhesion regions that promoted migration and cell adhesion. Molecular factors present in cementum have been proved to be important for development, maintenance and regeneration of cementum [5].

This study was conducted to investigate the expression and distribution of osteopontin on diseased root surface in comparison with healthy non- diseased root surface. To determine whether the amount and/or distribution of osteopontin are altered in teeth roots affected by periodontitis (diseased) by immunohistochemical technique using osteopontin mouse monoclonal antibody [NCL-O-PONTIN] (Novocastra) in relation to cemental surface, matrix and periodontal ligament.

\section{Materials and Methods}

\section{Collection of teeth}

Inclusion criteria: A Total of Fifty-five permanent tooth were collected from the Department of Oral Surgery. This included 25 permanent healthy human teeth, Impacted or partially erupted third molars and teeth requiring removal for orthodontic therapy and 30 permanent teeth affected by advanced periodontitis [grade III mobility], which was deemed hopeless. Prior to extraction, Periapical radiographs were taken to confirm the root completion of the impacted or partially erupted teeth. Immediately after extraction, the teeth were fixed in $10 \%$ neutral buffered formalin.

Exclusion criteria: Teeth which were exposed to previous root planning.

For this study age and type of periodontitis were not recorded because the study was based on the effect of attachment loss and exposure of root surface on the distribution of osteopontin. Informed consent was obtained from patients before extraction.

\section{Tooth preparation}

All teeth were initially fixed in $10 \%$ neutral buffered formalin. The teeth were then demineralized in buffered formic acid, which had a $\mathrm{pH}$ of 2.3.

Demineralization was confirmed radiographically after two weeks. The demineralized teeth were embedded in paraffin wax and Serial sections of $5 \mu \mathrm{m}$ were cut in a longitudinal plane and mounted on slides coated with $2 \% 3$-amino propyl triethoxysaline for Immunohistochemical staining. Additional sections were taken and stained with hemotoxylin and eosin (H\&E) for general histologic assessment.

\section{Immunohistochemistry}

\section{Reagents used were:}

1. Xylene

2. Alcohol $(90 \%, 70 \%)$

3. Citrate buffer (pH 6.2)

4. Phosphate buffered saline $(\mathrm{pH} 7.0)$

5. $3 \%$ hydrogen peroxide $\left(\mathrm{H}_{2} \mathrm{O}_{2}\right)$

6. Buffered casein solution

7. Primary Antibody-osteopontin mouse monoclonal antibody [NCL-O-PONTIN] (Novocastra)

8. Secondary Antibody - HorseRadish Peroxidase (poly-HRP) - conjugated goat anti-rabbit antibody (Bio Genex)

9. Normal rabbit serum (Bio Genex).

\section{Reagent preparation}

Primary antibody preparation: Antibody to osteopontin was diluted with phosphate buffered saline $(\mathrm{pH} 7.0)$ along with $1 \%$ bovine serum albumin in the ratio 1:100 (working dilution).

Secondary antibody preparation: HorseRadish Peroxidase (poly-HRP) - conjugated goat anti-rabbit antibody was diluted with Phosphate Buffered Saline containing 1\% Bovine Serum Albumin and 10\% Normal Human Serum in the ratio 1:100.

\section{Immunohistochemistry technique}

Tooth sections were deparaffinized in freshly prepared xylene and rehydrated in decreasing concentrations of alcohol, finally ending in Phosphate Buffered Saline. In order to expose the antigen sites that were blocked by formalin fixative, the slides were immersed in citrate buffer $(\mathrm{pH} \mathrm{6.2)}$ and placed in a microwave oven for 10 minutes. The endogenous peroxidase activity was inhibited by 10-minute exposure of the slides to $3 \%$ Hydrogen Peroxide. Blocking of non-specific binding was done by incubating the sections for 20 minutes in buffered casein solution with sodium azide (universal blocking agent) [6].

Two step immunoperoxidase method for the detection of osteopontin: The prepared primary antibody was applied to the sections and incubated for 60 minutes at $25^{\circ} \mathrm{C}$. The slides 
were washed three times for 5 minutes with phosphate buffered saline.

The sections were incubated after applying the prepared secondary antibody for one hour at $25^{\circ} \mathrm{C}$ and were again washed.

Horse Radish peroxidase was used as the substrate and Diaminobenzene (DAB) as the dye for 10 minutes. The slides were washed in distilled water. By using Harris hematoxylin solution, the stained sections were counter stained briefly. The slides were mounted using DPX medium and a coverslip. The slides were allowed to dry before viewing under light microscope. Photomicrographs were taken with a digital camera.

The above steps were repeated for all the sections using osteopontin mouse monoclonal antibody [NCL-O-PONTIN].

Controls: Control sections which included one healthy and one diseased tooth were treated in the same manner as the test groups except that the primary antibody was omitted and substituted with phosphate buffered saline and a non-immune antibody (Normal rabbit serum) at same concentration.

Assessment: Light microscopic assessments of the spatial distribution and localization of osteopontin antibodies were made in both healthy and diseased tooth sections. Three main areas were examined for antibody staining. These included the coronal third, middle third and apical third areas. For diseased teeth, a careful distinction was made between areas where cementum was not covered with periodontal ligament (exposed cementum) especially in coronal and middle third and areas at which overlying cementum was evident (nonexposed cementum).

\section{Results and Discussion}

A total of 55 collected teeth, which included 25 permanent healthy human teeth and 30 diseased permanent teeth were used to stain for osteopontin. Out of the 25 healthy teeth, 12 teeth (48\%) (Figure 1) and out of 30 diseased teeth 19 teeth (63\%) (Figure 2) were immunoreactive for osteopontin antibody. The remaining healthy and diseased teeth were excluded from the study because, they either stained negative for osteopontin antibody or displayed staining that was too non-specific for any meaningful analysis.

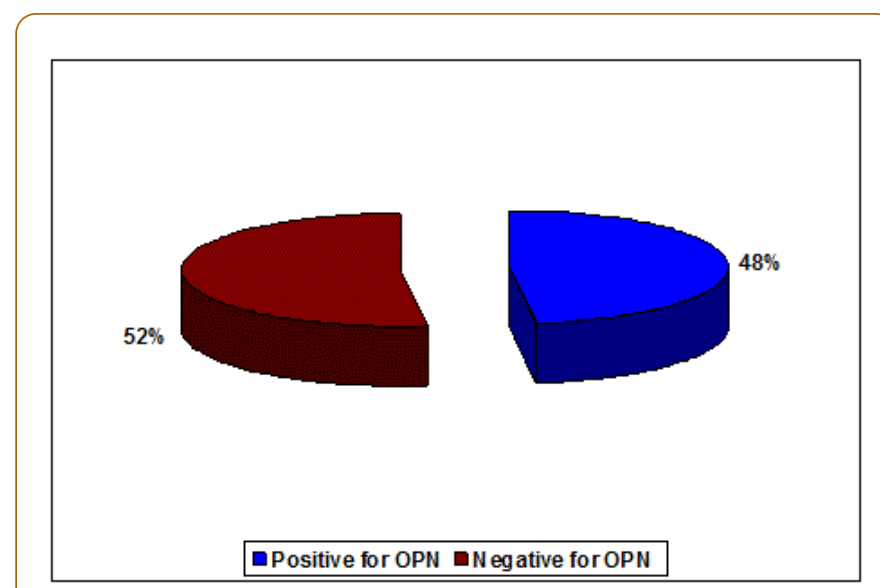

Figure 1 Immunoreactivity for Opn in Healthy Teeth.

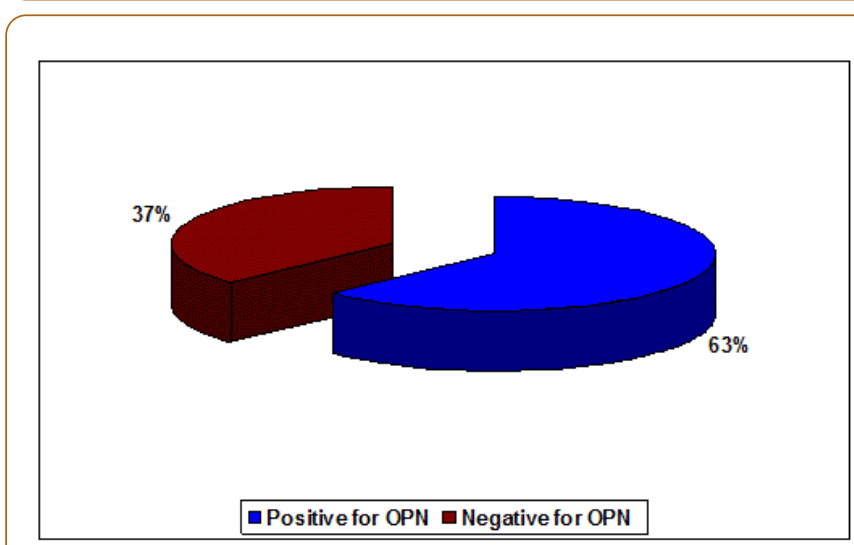

Figure 2 Immunoreactivity for Opn in Diseased Teeth.

The staining intensity was assessed in cemental matrix, cemental surface and periodontal ligament in coronal, middle and apical areas along the root surface. The sections which were included in the assessment were the ones where the staining for osteopontin antibody had taken up in the entire section. A Visual analog of stained pulp stones was taken as a guide for scoring the stain intensity (Figure $\mathbf{3}$ ). 
Out of 25 healthy tooth roots examined, $48 \%$ was positive for OPN. 52\% stained negative for OPN. $75 \%$ of the teeth from the sample teeth stained positive for OPN in coronal third, 83\% of the teeth stained positive for OPN in the middle third and $75 \%$ of the teeth stained positive for OPN in apical third (Table 1).

Figure 3 Visual Analog of Stained Pulp Stones.

Table 1 Summary of immunoreactivity and localization of osteopontin in healthy and diseased cementum.

\begin{tabular}{|c|c|c|c|c|c|c|c|c|c|c|c|}
\hline \multirow{2}{*}{ Bone OPN } & \multirow{2}{*}{$\begin{array}{l}\text { No of } \\
\text { Teeth }\end{array}$} & \multicolumn{2}{|c|}{ Positive for OPN } & \multicolumn{2}{|c|}{ Negative for OPN } & \multicolumn{2}{|c|}{$\begin{array}{l}\text { Coronal 3rd (Teeth } \\
\text { +ve for OPN) }\end{array}$} & \multicolumn{2}{|c|}{$\begin{array}{l}\text { Middle 3rd } \\
\text { +ve forOPN) }\end{array}$} & \multicolumn{2}{|c|}{$\begin{array}{l}\text { Apical 3rd (Teeth } \\
+ \text { ve for OPN) }\end{array}$} \\
\hline & & (No) & $(\%)$ & (No) & (\%) & (No) & $(\%)$ & (No) & $(\%)$ & (No) & $(\%)$ \\
\hline $\begin{array}{l}\text { Healthy(non- } \\
\text { exposed) }\end{array}$ & 25 & 12 & 48 & 13 & 52 & 9 & 75 & 10 & 83 & 9 & 75 \\
\hline Diseased (exposed) & 30 & 19 & 63 & 11 & 37 & 6 & 31 & 14 & 73 & 16 & 84 \\
\hline
\end{tabular}

Out of 30 teeth (diseased group), $63 \%$ of teeth was positive for OPN. $37 \%$ stained negative for OPN. $31 \%$ of teeth from the diseased group was positive for OPN in coronal third, $73 \%$ of the teeth stained positive for OPN in the middle third and $84 \%$ of the teeth stained positive for OPN in apical third (Figure 4).

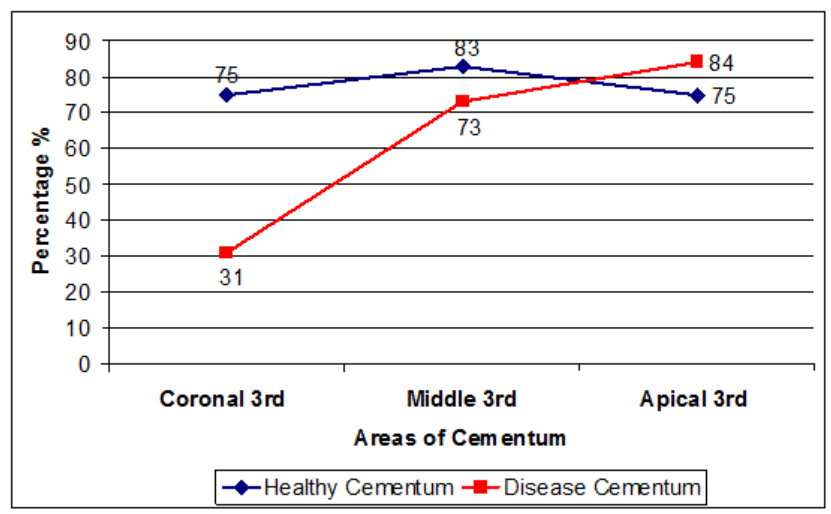

Figure 4 Immunolocalization of Opn in Healthy and Diseased Cementum.

\section{Immuno histochemical assessment of expression and distribution of opn}

Healthy teeth: In Coronal Third, Osteopontin Staining was mild in most of the sections along the cemental surface, adjacent to the periodontal ligament. There was mild to moderate staining of cemental matrix and moderate staining in the periodontal tissue. Staining of periodontal ligament cells for OPN was observed in close proximity to the cementum surface (Figure 5). In Middle Third, mild staining for Osteopontin in relation to the cemental surface and comparatively decreased intensity of staining in relation to cementum matrix was observed. Mild to moderate staining was observed in the periodontal ligament tissue (Figure 6). In Apical Third, mild staining was observed in the cemental matrix region. There was moderate localization of OPN along the cemental surface adjacent to the periodontal ligament. Mild to moderate staining was observed in the periodontal ligament tissue (Figure 7). 


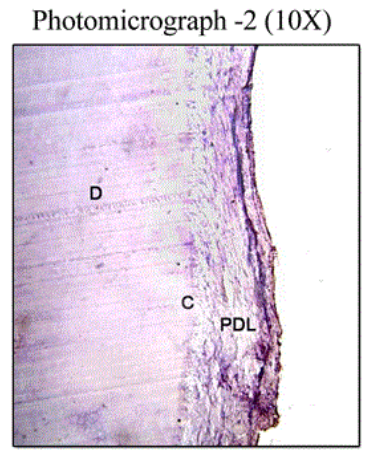

Photomicrograph - 4

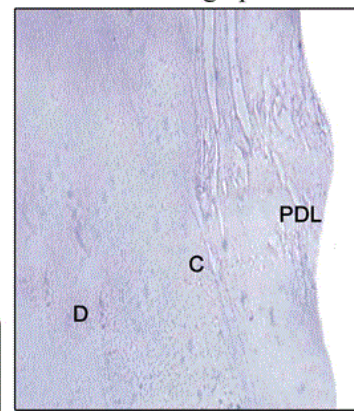

Photomicrograph -3 (40X)

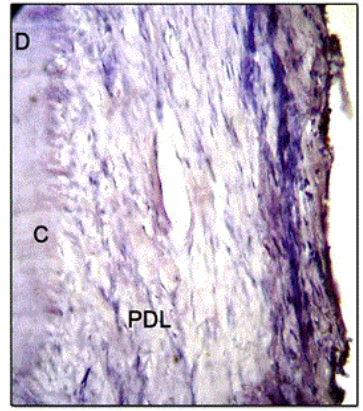

Figure 5 Opn Expressionin Coronal Third.

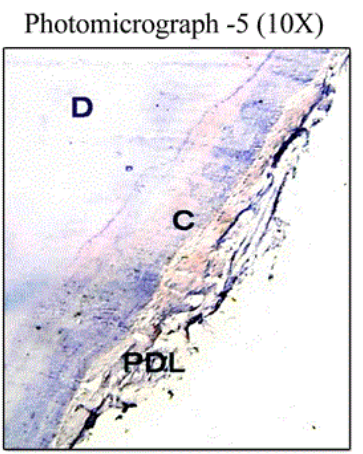

Photomicrograph - 7

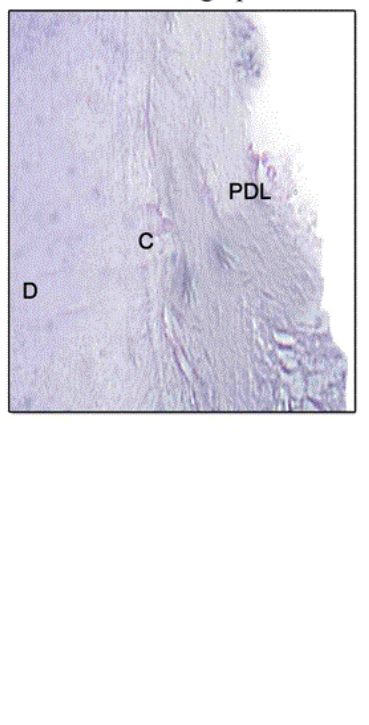

Figure 6 Opn Expression in Middle Third.

An intact periodontal ligament was observed in most of the areas of the root or cemental surfaces. Focal areas of staining in the periodontal ligament cells in close proximity to the cemental surface was observed. 


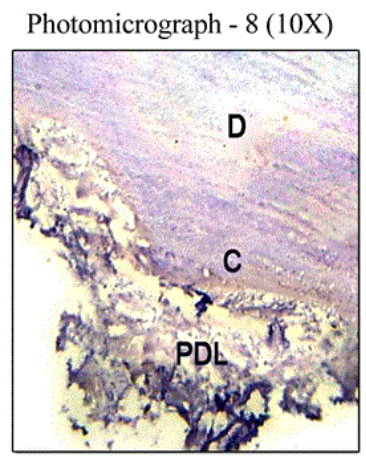

Photomicrograph - 10
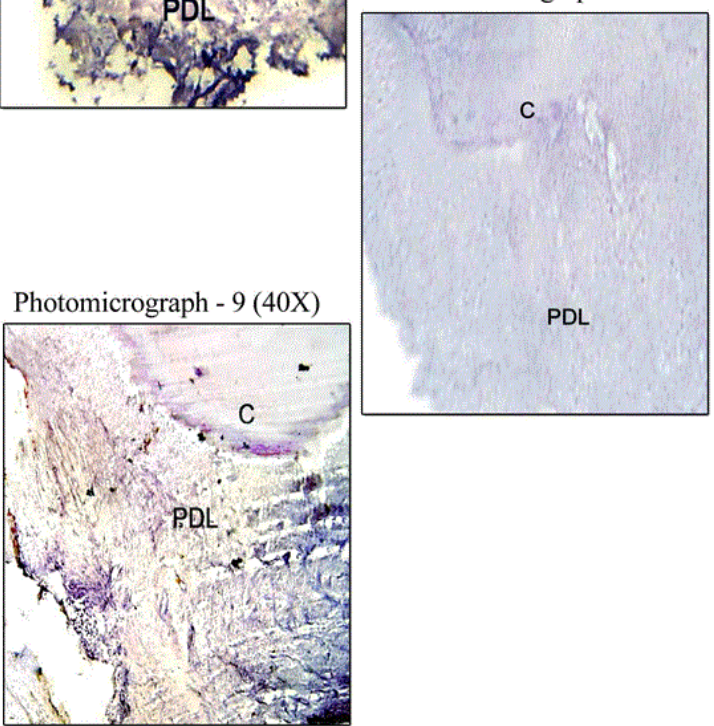

Figure 7 Opn Expression in Apical Third.

Diseased teeth: In Coronal Third Staining for Osteopontin in diseased teeth was minimal in areas where periodontal ligament was absent, i.e., where cementum was exposed, on comparison with the areas where the periodontal ligament was intact. Mild staining of Cemental matrix and mild to moderate staining of cemental surface was observed where the periodontal ligament was intact. The periodontal ligament matrix also showed moderate staining (Figure 8). In Middle Third, Mild to moderate staining for OPN along the cemental surface and cemental matrix adjacent to the periodontal ligament, with mild to moderate staining of the periodontal ligament tissue matrix was observed (Figure 9). In Apical Third, Strong staining for OPN was seen along the Cemental surface adjacent to the periodontal ligament. There was moderate staining of the periodontal ligament tissue matrix with strong staining of the periodontal ligament cells for OPN. Moderate staining for OPN were found in areas of hypercementosis adjacent to the periodontal ligament, with moderate staining of the cemental matrix. Central area of cemental matrix shows isolated regions of moderate staining (Figure 10).

\section{OPN EXPRESSION IN CORONAL THIRD}

Photomicrograph - 11 (10X)

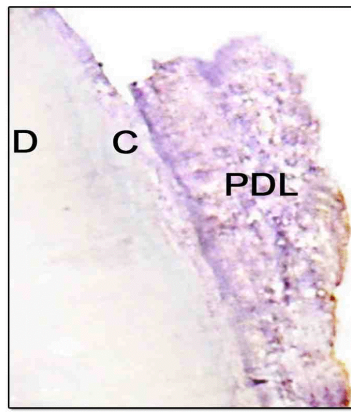

Photomicrograph - 13
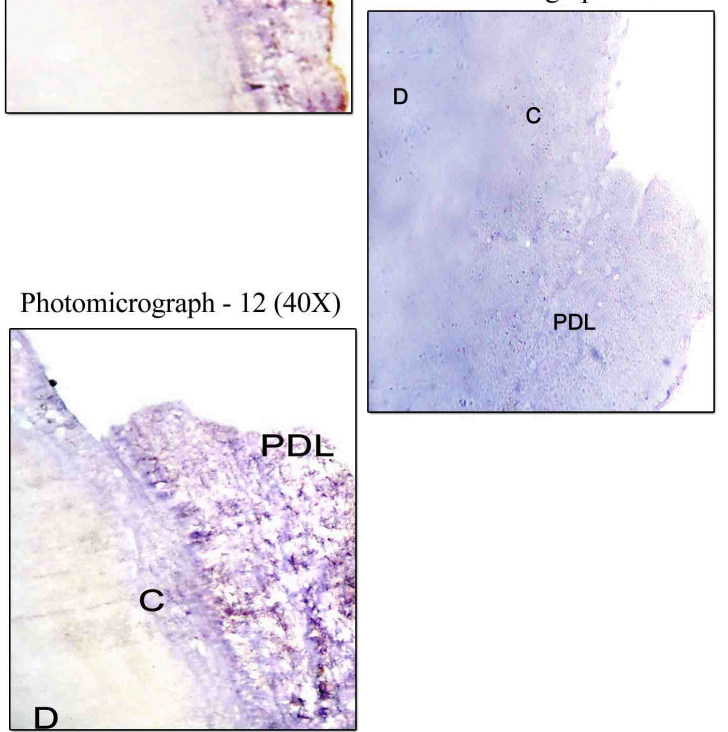

Figure 8 Opn Expressionin Coronal Third.

The control sections for the respective areas (coronal, middle and apical portions) are presented in photomicrograph $-4,7,10,13,16,19$. As this was a descriptive study of visual assessments of immunohistochemical staining, no statistical analysis of the data was performed.

An ultimate goal of periodontal regeneration therapy is to predictably restore tissues lost as a consequence of periodontal disease i.e., bone, cementum and periodontal ligament. Many molecular factors are responsible for the development, maintenance and regeneration of cementum. The proposed activity of the molecular factors includes adhesion or chemo-attraction, mitogenesis, differentiation and mineralization. 


\section{OPN EXPRESSION IN MIDDLE THIRD}

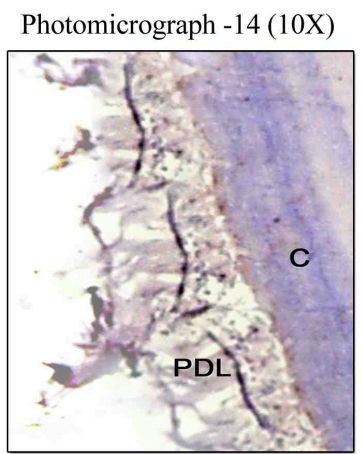

Photomicrograph - 16

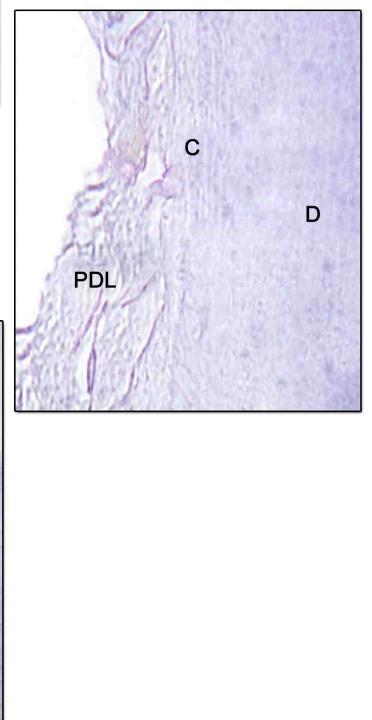

Figure 9 Opn Expressionin Middle Third.

Osteopontin (OPN) is a phosphorylated acidic glycoprotein synthesized by multiple cell types throughout the body in response to injury. In the tooth, OPN is expressed in bone and other mineralized connective tissue such as dentin and cementum. This non-collageneous protein is also expressed by cells like cementoblasts, osteoblasts and newly forming periodontal ligament cells. Osteopontin has a similar distribution pattern in bone and cementum. It fills in the interfibrillar spaces. Strong immunoreactivity is observed in acellular extrinsic fiber cementum because its matrix possesses large interfibrillar spaces. Recent studies have demonstrated that secretion of OPN following tooth transplantation, by immunocompetent cells plays a role in the differentiation of odontoblast-like cells resulting in reparative dentinogenesis, during pulpal healing [7].

\section{OPN EXPRESSION IN MIDDLE THIRD}

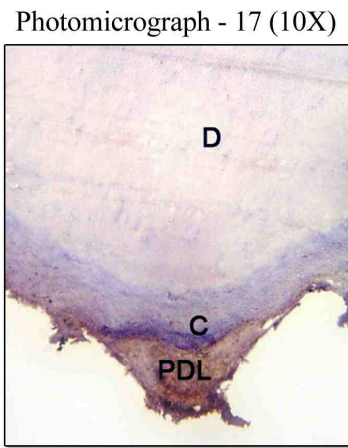

Photomicrograph -19

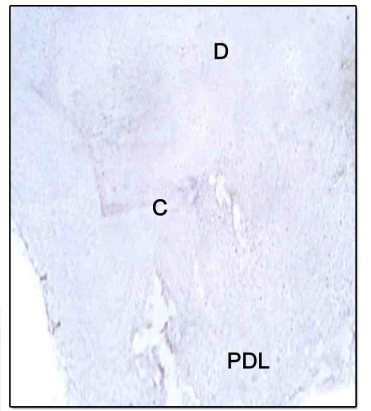

C

Photomicrograph - $18(40 \mathrm{X})$

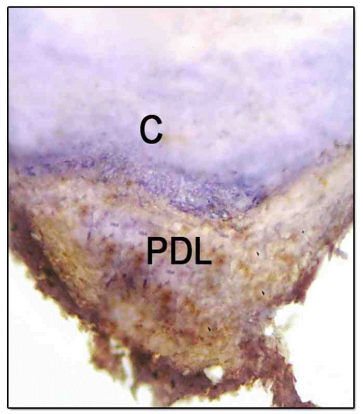

Figure 10 Opn Expressionin Middle Third.

The present study was done to determine the expression and distribution of osteopontin on the periodontally healthy human teeth, which included partially erupted or impacted third molar teeth, as Studies have demonstrated that periodontal ligament stem cells obtained from impacted and erupted tooth root had the same viability rate and teeth extracted for orthodontic reasons were further compared with the expression of OPN on periodontally diseased human tooth root [8]. Areas on the root surfaces examined for the expression of OPN were cemental matrix, cemental surface and periodontal ligament of periodontally involved and periodontally healthy human teeth.

Immunohistochemical analysis by using Osteopontin Mouse Monoclonal Antibody (NCL-O-PONTIN) (Novacastra) against human osteopontin (OPN), demonstrated the presence of OPN along the along cemental surface and in the periodontal ligament tissue of periodontally involved and periodontally sound human teeth.

Digital photomicroscopic assessment of spatial distribution and localization of OPN antibodies were made in three main areas along root surface i.e., coronal, middle and apical portion. 
In case of diseased teeth, distinction was made between the areas which were covered by PDL i.e., unexposed cementum and exposed cementum.

Out of 25 healthy teeth, 12 teeth (48\%) were immunoreactive for osteopontin and out of 30 diseased teeth, 19 teeth (63\%) were immunoreactive for osteopontin. The remaining samples which stained negative were excluded from the study. The amount of immunoreactivity obtained in this study was in corelation with the study done by Lao et al. [9].

In healthy teeth with intact PDL, the staining intensity for the distribution of OPN were found to be mild to moderate in cemental surface and matrix. OPN was observed to localize quite strongly in the periodontal ligament in this study. This corresponds to the fact that OPN is expressed by cells in a variety of soft and hard tissues. Thus, it indicates that OPN may have multiple functions depending on the tissue location [10].

Bosshardt et al. [11], demonstrated expression of OPN to be more in acellular afibrillar and acellular extrinsic fiber cementum fiber cementum compared to cellular intrinsic fibre cementum.

In present study, staining of PDL cells were observed in close proximity to the cemental surface, which was in accordance to the study done by Mac et al. [12]. This suggests that OPN plays a functional role in root and cementum development. OPN also plays a protective role during tooth development by acting as an inhibitor/regulator of mineralization.

Martin et al. [9], on comparing the spatial expression of Bone sialoprotein and Osteopontin in healthy and diseased root surface, demonstrated that OPN was observed to localize quite strongly in PDL, while bone sialoprotein appeared to be more specific to mineralized tissues.

Several studies have attempted to find differences between the cementum of healthy and periodontally involved teeth. No differences have been found in terms of surface characteristics, hardness, permeability and amino acid composition. Study done by Armitage et al. [13], demonstrated a hyper mineralized surface zone 10-20 micro meters wide in the cementum of exposed teeth.

In this study, pathological granules were found in the diseased cementum that was in accordance with the study done by Armitage et al. [13], where he did electron microscopic observation on exposed cementum and observed pathological granules in the cementum, which represented bacterially denatured collagen that picked up various substances from the oral environment. In all exposed specimen's dental calculus and microorganisms were associated with surface areas, whereas collagen fibers were observed in unexposed cementum.

The results in this study demonstrated that for diseased root there was absence of immunostaining for OPN in exposed cementum (absence of overlying PDL). This suggested that periodontally exposed root surfaces are exposed to agents from the oral environment that can lead to damage of the exposed cementum Which results in degradation of key proteins such as BSP and OPN from the cementum matrix.

Christgau et al. [14], determined the temporal and spatial expression pattern of extracellular matrix components in furcation defects, following guided tissue regeneration compared with open flap debridement. Following superficial resorption by osteopontin (OPN), osteoclasts and odontoclasts, cementum and bone formation ensued with strong expression of Bone sialoprotein (BSP) and osteopontin (OPN), along bone and tooth surfaces. Deposition of collagen-1, fibronectin, BSP, OPN, cementoid and osteoid became evident after 4 months. It was demonstrated that detrimental influence of inflammatory reaction caused bacterial contamination of exposed GTR membranes on the regeneration process. They inhibited or delayed the formation of collagen, as well as expression of BSP and OPN along the root surfaces, which seem to be crucial for new cementum formation.

In the apical part of the diseased tooth root, a strong staining for OPN was seen along the cementum surfaces adjacent to the periodontal ligament. This can be due to the presence of hyper mineralized zone of cementum in that area. There was a moderate staining of the periodontal ligament, and a strong staining of cells of periodontal ligament for OPN in areas of reparative cementum adjacent to the periodontal ligament with generalized light to moderate staining of the matrix of this cementum. A moderate staining for OPN was localized to periodontal ligament cells adjacent to reparative cementum. This was in accordance with findings of the study done by Martin et al. [9].

Studies demonstrated the expression of BSP and OPN in normal and healthy periodontal tissue, suggesting their important roles in healing, repair and regenerations [15-18].

Hasegawa et al. [19], noted that OPN expression by Epithelial cell rests of malassez(ERM) has been demonstrated in experimental cemental repair. The protein was expressed by ERM situated close to the cemental lacunae, hence it was speculated of a possible role of these cells, in mineralized tissue repair and cementum formation.

Fiorellini et al. [20], demonstrated the effect of Osteopontin and an Osteopontin-Derived Synthetic Peptide Coating on Osseointegration of Implants in a Canine Model, further suggested that capable of significantly accelerating the early stage of osseointegration and bone healing around implants.

Ideally the regenerated cementum should closely resemble the acellular extrinsic fiber cementum, because it contributes to the attachment function. In most periodontal regeneration studies, the quality of the attachment function is questionable, because the newly formed cementum is cellular, the numerical density of inserting fibers is low, and the interfacial bonding appears to be weak. Future experimental studies aimed at improving periodontal regeneration should mimic the natural root conditioning process to improve the interfacial bonding. 


\section{Conclusion}

Osteopontin Mouse Monoclonal Antibody (NCL-O-PONTIN) (Novacastra) with reliable supply of one pure antibody with known specificity was used to determine the staining for osteopontin (OPN). The absence of staining for OPN in all areas of exposed cementum (absence of overlying PDL) in diseased teeth can affect the ability for regeneration and new connective tissue attachment onto the previously denuded root surface. A knowledge of structure-function relationships of matrix interfaces is fundamental in order to develop more predictable regenerative therapies aimed at substituting pathologically altered mineralized tissues. Hence a better understanding of molecular biology gives us a better insight to what lies beneath and in improvising the therapeutic outcomes of regeneration.

\section{References}

1. Bartold PM, Sampath AN (1998) Biology of periodontal connective tissue. Quintessence Publishing.

2. D'Errico JA, Berry JE, Ouyang $\mathrm{H}$, Strayhorn $\mathrm{CL}$, Windle JJ, et al. (2000) Employing a transgenic animal model to obtain cementoblasts in vitro. J Periodontol 71: 63-72.

3. Nazan ES, William VG, Martha JS (2000) Molecular and cell biology of cementum. Periodontology 24: 73-98.

4. Lindskog S, Blomlof L (1983) Cementum hypoplasia in teeth affected by juvenile periodontitis. J Clin Periodontol 10: 443-451.

5. D'Errico JA, MacNeil RL, Takata T, Berry J, Strayhorn C, et al. (1997) Expression of bone associated markers by tooth root lining cells, in situ and in vitro. Bone 20: 117-126.

6. Bancroft JD, Gamble M (2008) Theory and practice of histological techniques. Elsevier Health Sciences.

7. Martin L, Victor M, Bartold PM (2006) Immunohistochemical study of bone sialoprotein and osteopontin in healthy and diseased root surfaces. J Peridontol 77: 1665-1673.

8. Saito K, Nakatomi M, Ida-Yonemochi H, Ohshima H (2016) Osteopontin Is Essential for Type I Collagen Secretion in Reparative Dentin. J Dent Res 95: 1034-1041

9. Dalband ML, Amiri I, Soltanian AR, Gholami M, Khayati A, et al. (2015) Comparing Viability of Periodontal Ligament Stem Cells
Isolated from Erupted and Impacted Tooth Root. J Craniofac Surg 26: e608-e612.

10. Sodek J, Chen J, Kasugai S, Nagata T (1992) Elucidating functions of the bone sialoproteins Osteopontin and bone sialoprotein. In: Slavkin H, Price PA (eds.) Chemistry and biology of Mineralized tissues. Elsevier, Amsterdam, pp: 297-307.

11. Bosshardt DD, Nanci A (1997) Immunodetection of enamel- and cementum-related (bone) proteins at the enamel-free area and cervical portion of the tooth in rat molars. J Bone Miner Res 12: 367-379.

12. MacNeil RL, Berry J, D'Errico J, Strayhorn C, Piotrowski B, et al. (1995) Role of two mineral-associated adhesion molecules, osteopontin and bone sialoprotein, during cementogenesis. Connect Tissue Res 33: 1-7.

13. Armitage GC, Christie TM (1973) Structural changes in exposed human cementum II. Electronmicroscopic observations. J Periodontal Res 8: 356-365.

14. Christgau M, Caffesse RG, Schmalz G, D'Souza RN (2007) Extracellular matrix expression and periodontal wound-healing dynamics following guided tissue regeneration therapy in canine furcation defects. J Clin Peridontol 34: 691-708.

15. Matsuura M, Herr Y, Han KY, Lin WL, Genco RJ, et al. (1995) Immunohistochemical expression of extracellular matrix components of normal and healing periodontal tissues in the beagle dog. J Periodontol 66: 579-593.

16. Amar S, Chung KM, Nam SH, Karatzas S, Myokai F, et al. (1997) Markers of bone and cementum formation accumulate in tissues regenerated in periodontal defects treated with expanded polytetrafluoroethylene membranes. J Periodontal Res 32: 148-158.

17. Ivanovski S, Li H, Haase HR, Bartold PM (2001) Expression of bone associated macromolecules by gingival and periodontal ligament fibroblasts. J Periodontal Res 36: 131-141.

18. Kawaguchi H, Ogawa T, Kurihara H, Nanci A (2001) Immunodetection of noncollagenous matrix proteins during periodontal tissue regeneration. J Periodontal Res 36: 205-213.

19. Hasegawa N, Kawaguchi H, Ogawa T, Uchida T, Kurihara H (2003) Immunohistochemical characteristics of epithelial cell rests of Malassez during cementum repair. J Periodontal Res 38: 51-56.

20. Fiorellini JP, Glindmann S, Salcedo J, Weber HP, Park CJ, et al. (2016) The Effect of Osteopontin and an Osteopontin-Derived Synthetic Peptide Coating on Osseointegration of Implants in a Canine Model. Int J Periodontics Restorative Dent 36: e88-e94. 